HCV genotype: G4 3/5 (60\%), G1 2/5 (40\%) (result of 1 patient, pending). Polymorphism of IL28B not favourable in 4/6: rs 12979860 CT and rs8099917 TG (2 patients pending genetic analysis). Fibroscan ${ }^{\circledR}$ at diagnosis: F2 3/6 (50\%), F0 (1), F1 (1), F3 (1). No patient showed jaundice as a clinical presentation. During the evolution nobody presented decline $\geq 2 \log$ of HCV-PCR at $1^{\text {st }}$ month of the diagnosis, neither on the $3^{\text {rd }}$ month spontaneous viral clearance. A patient has received treatment withpegIFN+ribavirine six months after the diagnosis, with rapid virological response (negative HCV-PCR at 4 wk).

Conclusion This report suggests that hepatitis $\mathrm{C}$ is an emergent STI in MSM population HIV-infected. The evolution towards chronicity is common. It should also be considered in case of sudden increase of transaminases, even without symptoms and therefore should be a part of the annual serology screening.

\section{P2.039 LATE PRESENTATION TO CARE REMAINS A PROBLEM IN CROATIAN NATIONWIDE COHORT}

doi:10.1136/sextrans-2013-051184.0304

I Grgic, L Gorenec, A Planinic, S Zidovec Lepej. University Hospital for Infectious Diseases, Zagreb, Croatia

Background Late presentation to care of HIV-positive individuals and late introduction of antiretroviral therapy can lead to occurance of opportunistic diseases and higher morbidity and mortality of patients. Croatia is a country with a low-level HIV epidemic. Even after interventions undertaken during the Croatian Global Fund Project in 2004-2006 late presentation to care remains a problem.

Methods The aim of this study was to determine the percentage of late presenters among newly-diagnosed HIV-positive individuals who entered clinical care from January of 2007 till December of 2011. Late presenters were defined as patients with < 350 CD4 T-cells per $\mu$ l. CD4 T-cell count was measured by flow cytometry (Beckman Coulter Flow Count reagent).

Results The number of patients diagnosed with HIV did not grow dramatically over the years (52 newly-diagnosed HIV-individuals entered clinical care in 2007, and 77 in 2010). The percentage of late presenters however, did grow over the years, from $46.2 \%$ in 2007 to $64 \%$ in 2011 . Still, the number of patients presented to care with less than $200 \mathrm{CD} 4 \mathrm{~T}$-cells/ $\mu$ l was the lowest in 2011 (30 patients out of $48,62.5 \%$ ), and highest in 2007 (19 out of 24 patients, $79.2 \%$ ).

Conclusion The percentage of late presenters in Croatia is still quite high, even though there are fewer patients with less than 200 CD4 T-cells $/ \mu$ l. A national strategy for earlier entrance to care should be developed.

\section{P2.040 MULTIPLEXED FLUORESCENCE IMMUNOASSAY SYSTEM FOR RAPID SEROLOGIC TESTING AT THE POINT-OF-CARE}

doi:10.1136/sextrans-2013-051184.0305

M Lochhead, K Todorof, J Ives, C Myatt. MBio Diagnostics, Inc., Boulder, CO, United States

Background MBio Diagnostics is developing a multiplexed immunoassay platform capable of simultaneous detection of serologic disease markers from a single drop of blood. Here we demonstrate the system in the context of HIV and AIDS-related co-infection testing. Multianalyte testing at the time of HIV diagnosis is essential for individualised management of HIV infection. The MBio System is designed to address the unmet need for timely and cost-effective co-infection testing.

Methods The MBio multiplexed immunoassay system is based on single-use disposable cartridges and an inexpensive reader. A simple, 10 minute assay protocol was developed for delivering HIV-1 antibody $(\mathrm{Ab})$ reactivity results on whole blood, plasma, or serum samples. A total of 87 whole blood samples were run with the 10 minute assay. $50 \mathrm{HIV}-1 \mathrm{Ab}$ negative samples were used to establish cutoffs. $37 \mathrm{HIV}-1 \mathrm{Ab}$ positive samples were used to assess system sensitivity. A set of 5 commercially available HIV-1 seroconversion panels were also used to assess the system. System demonstration in the context of syphilis and hepatitis $\mathrm{C}$ virus (HCV) testing was also performed on a subset of clinical specimens.

Results Ab reactivity results using the 10 minute assay protocol showed $100 \%$ concordance with known HIV serostatus for the 87 whole blood samples tested. Data for the seroconversion panels showed that MBio System performance meets or exceeds package insert data for FDA-approved HIV Ab rapid diagnostic tests. Simultaneous detection of syphilis (T. pallidum) and HCV Ab reactivity has been demonstrated.

Conclusions The dataset presented here demonstrates a simple, 10 minute assay protocol on the MBio multiplexed immunoassay system. Multianalyte testing from unprocessed whole blood at the POC should enable improved therapeutic decision making, particularly in limited resource settings.

\section{P2.041 INTRODUCING A NEW TYPE OF HIV RAPID TESTING BASED ON ORAL FLUID AT NON-GOVERNMENTAL ORGANISATIONS OF KYRGYZ REPUBLIC}

doi:10.1136/sextrans-2013-051184.0306

D Saliev. GFATM/UNDP Grants Implementation Unit, Bishkek, Kyrgyzstan

Background In Kyrgyz Republic HIV mainly spreads among the high risk behaviour population groups (mainly injection drug users - IDUs). Approximately 450,000 people are being tested for HIV in Kyrgyzstan per annum and less than $1 \%$ of them are IDUs, when testing of IDUs results in more than $60 \%$ of all new infections. Thus, access to testing remains the main challenge and priority for the national response to HIV.

Methods The UNDP in Kyrgyzstan, jointly with the Republican AIDS centre have started a roll out of HIV rapid testing based on oral fluid. For this pilot, there've been assessed and selected 12 nongovernmental organisations (NGOs), who work with IDUs, sex workers and men who have sex with men. A pool of non-medical testing counsellors was certified after trainings on rapid testing based on CDC/WHO training modules. OraQuick Advanced HIV 1-2 rapid test was selected for the roll out of the pilot.

Results Within the first three months of the pilot, 1,335 clients of the mentioned above NGOs, were tested for HIV by using oral fluid rapid tests. Some $6 \%$ of tested, had preliminary positive results of rapid test and were referred to nearest AIDS centres for further HIV confirmatory tests (ELIZA, Western Blot). There were only 2 cases of false positive results of rapid tests, which is less than $0.15 \%$ of all rapid tests results.

Conclusions Kyrgyz Republic is the first country in the Central Asian region, who introduced this new type of HIV rapid testing at community based organisations. First few months of the pilot have shown that non-medical professionals can provide this type of services to their clients, after the proper training. Now, people from the high risk behaviour population groups, especially those that had never been tested for HIV, are being tested at NGOs with rapid tests.

\section{P2.042 AN AUDIT OF HIV TESTING RATES IN PATIENTS ADMITTED WITH PNEUMONIA PRE- AND POST- IMPLEMENTATION OF OPT-OUT HIV TESTING FOR ACUTE MEDICAL ADMISSIONS}

doi:10.1136/sextrans-2013-051184.0307

E Wallis, J Saunders, C Orkin. Barts Health NHS Trust, London, UK

Background UK National Guidelines for HIV Testing recommend that an HIV test should be considered in all general medical 